\title{
Excited States of Positronic Lithium and Beryllium
}

\begin{abstract}
Sergiy Bubin and Oleg V. Prezhdo
Department of Chemistry, University of Rochester, Rochester, New York 14627, USA (Received 31 July 2013; revised manuscript received 19 September 2013; published 4 November 2013)

Using a variational method with an explicitly correlated Gaussian basis, we study the $e^{+}-\mathrm{Li}$ and $e^{+}-\mathrm{Be}$ complexes in the ground and lowest excited states with higher spin multiplicity. Our calculations provide rigorous theoretical confirmation that a positron can be attached to the excited states: $1 s 2 s 2 p^{4} P^{o}$ and $1 s^{2} 2 s 2 p^{3} P^{o}$ for $e^{+}$-Li and $e^{+}$-Be, respectively. The result is particularly notable for the $e^{+}$-Be complex, as the excited ${ }^{3} P^{o}$ state lies below the autoionization threshold. We report accurate binding energies, annihilation rates and structural properties of these positron-atom systems. The existence of the ground and metastable excited states with bound positron opens up a new route to the presently lacking experimental verification of stability of a positron binding to any neutral atom.
\end{abstract}

Understanding the mechanisms of the interaction of low energy positrons with matter is one of the main tasks of positron physics and chemistry. In particular, of great interest is the question whether atoms and molecules can capture positrons and form bound states stable against dissociation [1-9]. Since 1997, when the first conclusive and rigorous theoretical confirmation of a possibility of attaching a positron to a neutral lithium was given [1], there have been a number of investigations claiming the dynamical stability of positron-atom complexes. At present, at least a dozen atoms are believed to be capable of binding a positron. In contrast, on the experimental side no evidence has been collected as of yet to demonstrate the existence of positronic atoms. While different experimental approaches to study positron binding to atoms have been proposed, e.g., by measuring resonant positron-atom annihilation $[10,11]$ or by laser-assisted photorecombination [12], the existence of excited states is of crucial importance for detecting positron-atom complexes as it should allow spectroscopic measurements. Just recently such an approach has been used by Cassidy et al. [13] to confirm the production of a positronium molecule $\left(\mathrm{Ps}_{2}\right)$. The technique employed in Ref. [13] was based on observing small yet detectable changes in the annihilation yield of dense Ps over a narrow range of wavelengths corresponding to a transition between the ground and excited states of $\mathrm{Ps}_{2}$. The existence of a bound excited state of $\mathrm{Ps}_{2}$, in turn, had been previously predicted by numerical calculations [14].

Only a handful of theoretical studies so far have dealt with the investigation of excited states of positronic atoms. The simplest multielectron atom, helium, has been known to attach a positron in its $1 s 2 s^{3} S$ state for more than a decade [15] (here and below the term symbol refers to electrons only). Recently, it has also been shown that positron attachment is possible in three doubly excited states [16-18]. Nevertheless, in its ground singlet state He does not form a bound positron-atom complex. There was also an indication based on large configuration interaction $(\mathrm{CI})$ calculations that $\mathrm{Ca}$ and $\mathrm{Be}$ might also bind a positron in an excited state [6,19]. These calculations, however, did not yield positive binding energy values. Instead, they relied heavily on asymptotic series analysis and extrapolation, and involved a fixed-core approximation. It is only recently that Bressanini [9] demonstrated convincingly that lithium can attach a positron in the excited $1 s 2 s 2 p^{4} P^{o}$ state. While an encouraging fact by itself, it is somewhat unfortunate that this quartet state of $\mathrm{Li}$ lies far above the autoionization threshold. Its lifetime on the order of microseconds [20] is primarily determined by the relatively quick autoionization process. The question of the existence of positron-atom complexes in metastable states, whose energies are below the autoionization threshold, is much more intriguing from the practical point of view. In this Letter, we report a rigorous confirmation of such possibility for the $1 s^{2} 2 s 2 p^{3} P^{o}$ state of a Be atom (see Fig. 1). The lowest excited state of Be, it can only decay radiatively to the ground ${ }^{1} S$ state by emitting two or more photons. Its lifetime exceeds $2 \mathrm{~s}$ [21]. Our calculations allowed us to estimate also the lifetimes of both $e^{+}-\mathrm{Li}$

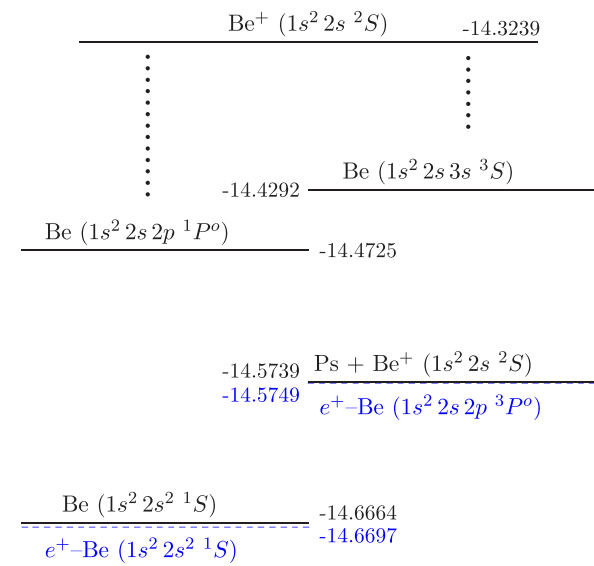

FIG. 1 (color online). Energy levels of $\mathrm{Be}$ and $\mathrm{Be}^{+}$(solid black lines) and $e^{+}$-Be (dashed blue lines). 
and $e^{+}-\mathrm{Be}$ complexes against electron-positron annihilation.

An accurate description of positronic systems is challenging even in their ground states. The difficulties stem from the presence of a different kind of light particle and weak binding energies. Moreover, due to strong repulsion from nuclei, the positron transforms the system into a clusterlike structure, which may result in poor convergence of traditional quantum-chemical approaches [22,23]. Explicitly correlated Gaussian (ECG) basis sets that depend on all interparticle distances provide effective means for overcoming these obstacles for small systems. For the case of $L=0,1$ considered in this work, where $L$ is the total orbital angular momentum, a suitable form of the spatial part of ECGs is

$$
\begin{gathered}
\phi_{k}^{(L=0)}=\exp \left[-\sum_{i} \alpha_{i k} r_{i}^{2}-\sum_{i<j} \beta_{i j k} r_{i j}^{2}\right], \\
\phi_{k}^{(L=1, M=0)}=z_{p_{k}} \exp \left[-\sum_{i} \alpha_{i k} r_{i}^{2}-\sum_{i<j} \beta_{i j k} r_{i j}^{2}\right],
\end{gathered}
$$

where $\mathbf{r}_{i}$ are particle coordinates, $r_{i j}=\left|\mathbf{r}_{i}-\mathbf{r}_{j}\right|$, and $\alpha_{i k}$, $\beta_{i j k}$, and $p_{k}$ are variational parameters. The explicit use of the spin part of the wave function can be avoided by employing Young projection operators within spin-free formalism [24]. Variational expansions in terms of ECGs have demonstrated an exceptional performance in calculations of various quantum few-body systems, including those containing positrons [25-28].

It has been known that the condition and likelihood of positron binding to an atom $A$ depends on the ionization potential (IP) of the atomic state [5]. When the IP is greater than 0.25 hartree (binding energy of Ps), the threshold for the total energy is set by dissociation channel $e^{+}+\mathrm{A}$. In this case the key property affecting the existence of a bound state is the atomic polarizability. When the IP is smaller than 0.25 , the dissociation channel $\mathrm{Ps}+\mathrm{A}^{+}$ becomes more competitive. It has been observed that a positron is more likely to form a bound state with an atom when the IP value is not far from 0.25 . The ground and excited states of $\mathrm{Li}$ and $\mathrm{Be}$ considered in this Letter satisfy that condition fairly well, as demonstrated in Table I. Regardless of the dissociation threshold, a bound

TABLE I. Atomic ionization potentials (in hartree, from Ref. [29]) and dissociation thresholds for positron-atom complexes.

\begin{tabular}{lcc}
\hline \hline$e^{+}-\mathrm{A}($ state $)$ & IP[A(state) $)$ & Dissociation threshold \\
\hline$e^{+}-\mathrm{Li}\left(1 s^{2} 2 s^{2} S\right)$ & 0.198 & $\operatorname{Ps}+\mathrm{Li}^{+}\left(1 s^{2}{ }^{1} S\right)$ \\
$e^{+}-\mathrm{Li}\left(1 s 2 s 2 p^{4} P^{o}\right)$ & 0.255 & $e^{+}+\operatorname{Li}\left(1 s 2 s 2 p^{4} P^{o}\right)$ \\
$e^{+}-\operatorname{Be}\left(1 s^{2} 2 s^{2}{ }^{1} S\right)$ & 0.343 & $e^{+}+\operatorname{Be}\left(1 s^{2} 2 s^{2}{ }^{1} S\right)$ \\
$e^{+}-\mathrm{Be}\left(1 s^{2} 2 s 2 p^{3} P^{o}\right)$ & 0.242 & $\operatorname{Ps}+\mathrm{Be}^{+}\left(1 s^{2} 2 s^{2} S\right)$ \\
\hline \hline
\end{tabular}

positron-atom state can be generally viewed as a mixture of two major configurations involving different binding mechanisms, namely, a positron interacting with a polarized atom and a polarized Ps atom interacting with an atomic ion.

The convergence of the total nonrelativistic energies for the $e^{+}-\mathrm{Li}$ and $e^{+}-\mathrm{Be}$ positronic complexes and relevant states of the atoms and ions is shown in Table II. We performed calculations using finite masses for the atomic nuclei: $M\left({ }^{7} \mathrm{Li}\right)=12786.3933 m_{e}$ and $M\left({ }^{9} \mathrm{Be}\right)=$ $16424.2037 m_{e}$, where $m_{e}$ is the electron mass. To enable direct comparison with energies from published works we also recalculated all quantities by setting the nuclear mass to infinity.

TABLE II. Convergence of total nonrelativistic energies and

\begin{tabular}{|c|c|c|c|c|}
\hline Basis size & Nucleus & & & $\mathrm{BE}$ \\
\hline & & $\mathrm{Li}^{+}\left({ }^{1} S\right)$ & $e^{+}-\operatorname{Li}\left({ }^{2} S\right)$ & \\
\hline 500 & ${ }^{7} \mathrm{Li}$ & -7.27932152 & -7.53173145 & 0.002410 \\
\hline 1000 & ${ }^{7} \mathrm{Li}$ & -7.27932152 & -7.53180242 & 0.002481 \\
\hline 1500 & ${ }^{7} \mathrm{Li}$ & -7.27932152 & -7.53181166 & 0.002490 \\
\hline 2000 & ${ }^{7} \mathrm{Li}$ & -7.27932152 & -7.53181432 & 0.002493 \\
\hline 2500 & ${ }^{7} \mathrm{Li}$ & -7.27932152 & -7.53181532 & 0.002494 \\
\hline 3000 & ${ }^{7} \mathrm{Li}$ & -7.27932152 & -7.53181573 & 0.002494 \\
\hline 3000 & ${ }^{\infty} \mathrm{Li}$ & -7.27991341 & -7.53241048 & 0.002497 \\
\hline \multirow[t]{2}{*}{ Ref. $[30]^{a}$} & ${ }^{\infty} \mathrm{Li}$ & -7.2799134 & -7.5323955 & 0.002482 \\
\hline & & $\operatorname{Li}\left({ }^{4} P^{o}\right)$ & $e^{+}-\mathrm{Li}\left({ }^{4} P^{o}\right)$ & \\
\hline 500 & ${ }^{7} \mathrm{Li}$ & -5.36760551 & -5.37319950 & 0.005594 \\
\hline 1000 & ${ }^{7} \mathrm{Li}$ & -5.36760579 & -5.37338670 & 0.005781 \\
\hline 1500 & ${ }^{7} \mathrm{Li}$ & -5.36760581 & -5.37341778 & 0.005812 \\
\hline 2000 & ${ }^{7} \mathrm{Li}$ & -5.36760582 & -5.37342757 & 0.005822 \\
\hline 2500 & ${ }^{7} \mathrm{Li}$ & -5.36760582 & -5.37343152 & 0.005826 \\
\hline 3000 & ${ }^{7} \mathrm{Li}$ & -5.36760582 & -5.37343343 & 0.005828 \\
\hline 3000 & ${ }^{\infty} \mathrm{Li}$ & -5.36801015 & -5.37383548 & 0.005825 \\
\hline \multirow[t]{2}{*}{ Ref. [9] ${ }^{\mathrm{b}}$} & ${ }^{\infty} \mathrm{Li}$ & $-5.36733(3)$ & $-5.3710(2)$ & $0.0037(2)$ \\
\hline & & $\operatorname{Be}\left({ }^{1} S\right)$ & $e^{+}-\operatorname{Be}\left({ }^{1} S\right)$ & \\
\hline 500 & ${ }^{9} \mathrm{Be}$ & -14.66642842 & -14.66915313 & 0.002725 \\
\hline 1000 & ${ }^{9} \mathrm{Be}$ & -14.66643460 & -14.66955355 & 0.003119 \\
\hline 1500 & ${ }^{9} \mathrm{Be}$ & -14.66643525 & -14.66963347 & 0.003198 \\
\hline 2000 & ${ }^{9} \mathrm{Be}$ & -14.66643537 & -14.66966210 & 0.003227 \\
\hline 2500 & ${ }^{9} \mathrm{Be}$ & -14.66643547 & -14.66967434 & 0.003239 \\
\hline 2500 & ${ }^{\infty} \mathrm{Be}$ & -14.66735645 & -14.67059286 & 0.003236 \\
\hline \multirow[t]{2}{*}{ Ref. [31] ${ }^{c}$} & ${ }^{\infty} \mathrm{Be}$ & -14.667338 & -14.670519 & 0.003181 \\
\hline & & $\mathrm{Be}^{+}\left({ }^{2} S\right)$ & $e^{+}-\operatorname{Be}\left({ }^{3} P^{o}\right)$ & \\
\hline 500 & ${ }^{9} \mathrm{Be}$ & -14.32386315 & -14.57277452 & -0.001089 \\
\hline 1000 & ${ }^{9} \mathrm{Be}$ & -14.32386347 & -14.57426328 & 0.000400 \\
\hline 1500 & ${ }^{9} \mathrm{Be}$ & -14.32386349 & -14.57462984 & 0.000766 \\
\hline 2000 & ${ }^{9} \mathrm{Be}$ & -14.32386349 & -14.57478465 & 0.000921 \\
\hline 2500 & ${ }^{9} \mathrm{Be}$ & -14.32386349 & -14.57486380 & 0.001000 \\
\hline 2500 & ${ }^{\infty} \mathrm{Be}$ & -14.32476318 & -14.57576495 & 0.001002 \\
\hline
\end{tabular}
positron binding energies (BE). All values are in hartree.

${ }^{\mathrm{a}} \mathrm{ECG}, 1200$ basis functions.

${ }^{b}$ Fixed-node diffusion Monte Carlo simulations.

${ }^{\mathrm{c}} \mathrm{ECG}, 2200$ basis functions. 
The data in Table II exhibit excellent agreement with and marginal improvement over the best previous calculations of the ground states of $e^{+}-\mathrm{Li}$ and $e^{+}$-Be by Mitroy et al. [30,31]. We have confirmed the stability of the excited $1 s 2 s 2 p^{4} P^{o}$ state of positronic lithium, and significantly increased the accuracy of the binding energy from 0.0037 hartree reported in Ref. [9] to $0.0058276(10)$ hartree obtained in this work. Finally, our efforts to compute the $1 s^{2} 2 s 2 p^{3} P^{o}$ state of $e^{+}$-Be have provided conclusive evidence that beryllium can attach a positron in an excited triplet state. The positron binding energy in that state is rather small and equal to just $0.0010003(400)$ hartree or $0.02722(10) \mathrm{eV}$. Because of tighter energy convergence in the case of bare atoms in comparison with the corresponding positron-atom complexes, our calculated binding energies are undoubtedly bounds from below to the exact nonrelativistic values. The uncertainties are estimated by extrapolating to the limit of the infinite basis size. Given the sufficiently high convergence of the total energies and no approximations involved in the calculations (at the nonrelativistic level), the prediction of the existence of the bound excited state is rigorous. The inclusion of relativistic corrections would not alter this conclusion, as they cancel out to a large extent in weakly bound systems when the energy difference (e.g., $e^{+}$-A vs Ps $+\mathrm{A}^{+}$) is evaluated. This happens because the largest contribution to the relativistic correction is due to core electrons, which remain essentially undistorted upon binding a positron. Rough estimates suggest that the net effect of relativistic corrections on the positron binding energy in the $1 s^{2} 2 s 2 p^{3} P^{o}$ state of $e^{+}-\mathrm{Be}$ is at least 1-2 orders of magnitude smaller than the binding energy itself. We have computed the change in the binding energy due to the inclusion of the two largest corrective terms, the mass velocity and Darwin terms, and obtained a shift of only 0.000007 hartree.

Table III lists expectation values, which allow us to compare the structure of the excited states of positronic lithium and beryllium with that of the ground states as well as with the related atoms and ions. As a result of weak binding, positronic atoms typically have a very large spatial extent and possess halolike properties [32]. Remarkably, for both $e^{+}$- $\mathrm{Li}$ and $e^{+}-\mathrm{Be}$, the size of the system as a whole in the excited state is smaller than in the ground state. The value of the average nucleus-positron distance, $\left\langle r_{n e^{+}}\right\rangle$, drops notably in the exited states. This fact can be rationalized in the case of Li by the almost twice higher binding energy in the $1 s 2 s 2 p^{4} P^{o}$ state compared to the $1 s^{2} 2 s^{2} S$ state. For Be, however, where binding in the excited state is extremely weak, the exact reason for such behavior is not immediately clear. The atomic ${ }^{3} P^{o}$ state, i.e., when no positron is attached, is predictably larger than the ground ${ }^{1} S$ state. The near equality of $\left\langle r_{n e^{+}}\right\rangle$with $\left\langle r_{e^{+}} e^{-}\right\rangle$ leads to the conclusion that $e^{+}-\mathrm{Be}\left(1 s^{2} 2 s^{2}{ }^{1} S\right)$ fits well into the picture of a bare positron interacting with a distant atom. This conclusion becomes even more evident when comparing nucleus-electron and electron-electron pair correlation functions, $g_{i j}(\mathbf{r})=\left\langle\delta\left(\mathbf{r}_{i j}-\mathbf{r}\right)\right\rangle$, shown in Fig. 2 . The change in these two distributions upon going from the $\mathrm{Be}$ atom to the $e^{+}$-Be complex is tiny. In contrast, the same two distributions, $g_{n e^{-}}(\mathbf{r})$ and $g_{e^{-}} e^{-}(\mathbf{r})$, exhibit noticeable difference between $\operatorname{Li}\left(1 s^{2} 2 s^{2} S\right)$ and $e^{+}-\operatorname{Li}\left(1 s^{2} 2 s^{2} S\right)$. The ratio between $\left\langle r_{n e^{+}}\right\rangle$and $\left\langle r_{e^{+}} e^{-}\right\rangle$for $e^{+}-\operatorname{Li}\left(1 s^{2} 2 s^{2} S\right)$ suggests that this system can be mainly viewed as a weakly distorted Ps- $\mathrm{A}^{+}$complex.

The geometric structure interpretation for the excited $P$ states of positronic atoms is somewhat less straightforward. The value of $\left\langle r_{n e^{+}}\right\rangle$is larger for $e^{+}$-Be compared to $e^{+}$-Li. At the same time the average positron-electron distance, $\left\langle r_{e^{+}} e^{-}\right\rangle$, is smaller. While the ratio between the $\left\langle r_{n e^{+}}\right\rangle$and $\left\langle r_{e^{+} e^{-}}\right\rangle$in the $1 s^{2} 2 s 2 p^{3} P^{o}$ state of $e^{+}$-Be is not exactly consistent with a Ps- ${ }^{+}$configuration, the positron-electron correlation function shown in Fig. 3 has a pronounced maximum at small positron-electron separation, which suggests that this configuration dominates.

TABLE III. Expectation values (in atomic units) for the ground and excited states of positronic complexes and relevant atoms and ions. For convenience, we use $n, e^{+}$, and $e^{-}$as indices, to denote nucleus, positron, and electron respectively. $\Gamma$ is the spin-averaged annihilation rate (in $10^{9} \mathrm{~s}^{-1}$ ). All quantities were computed using the largest generated ECG basis. Values in parentheses show estimated remaining uncertainty due to finite size of the basis.

\begin{tabular}{|c|c|c|c|c|c|c|c|c|c|}
\hline System & $\left\langle r_{n e^{-}}\right\rangle$ & $\left\langle r_{e^{-}} e^{-}\right\rangle$ & $\left\langle r_{n e^{+}}\right\rangle$ & $\left\langle r_{e^{+} e^{-}}\right\rangle$ & $\left\langle\delta\left(\mathbf{r}_{n e^{-}}\right)\right\rangle$ & $\left\langle\delta\left(\mathbf{r}_{e^{-}} e^{-}\right)\right\rangle$ & $\left\langle\delta\left(\mathbf{r}_{n e^{+}}\right)\right\rangle$ & $\left\langle\delta\left(\mathbf{r}_{e^{+} e^{-}}\right)\right\rangle$ & $\Gamma$ \\
\hline Ps & 3.000000 & & & & & & & 0.039789 & 2.0028 \\
\hline $\mathrm{Li}^{+}\left({ }^{1} S\right)$ & $0.572821(0)$ & $0.862373(0)$ & & & $6.85035(3)$ & $0.533608(6)$ & & & \\
\hline $\operatorname{Li}\left({ }^{2} S\right)$ & $1.663312(0)$ & $2.889697(0)$ & & & $4.61304(5)$ & $0.181404(10)$ & & & \\
\hline$e^{+}-\operatorname{Li}\left({ }^{2} S\right)$ & $3.4112(4)$ & $6.3635(8)$ & $9.9476(11)$ & $7.7773(8)$ & $4.5706(15)$ & $0.17843(10)$ & $0.0000018(2)$ & $0.011582(20)$ & $1.7484(30)$ \\
\hline $\operatorname{Li}\left({ }^{4} P\right)$ & $2.034578(0)$ & $3.495662(0)$ & & & $2.978053(50)$ & $0(0)$ & & & \\
\hline$e^{+}-\operatorname{Li}\left({ }^{4} P\right)$ & $2.27248(8)$ & $3.91373(15)$ & $9.0956(8)$ & $8.8956(8)$ & $2.9780(20)$ & $0(0)$ & $0.0000019(3)$ & $0.003941(10)$ & $0.59511(150)$ \\
\hline $\mathrm{Be}^{+}\left({ }^{2} S\right)$ & $1.033863(0)$ & $1.755787(0)$ & & & $11.69922(30)$ & $0.526780(40)$ & & & \\
\hline $\operatorname{Be}\left({ }^{1} S\right)$ & $1.493194(0)$ & $2.545443(0)$ & & & $8.83948(15)$ & $0.26756(4)$ & & & \\
\hline$e^{+}-\mathrm{Be}\left({ }^{1} S\right)$ & $1.53573(2)$ & $2.60626(4)$ & $9.9998(35)$ & $9.9575(35)$ & $8.819(15)$ & $0.2676(4)$ & $0.0000025(10)$ & $0.002140(10)$ & $0.43095(200)$ \\
\hline $\operatorname{Be}\left({ }^{3} P\right)$ & $1.556996(1)$ & $2.693011(2)$ & & & $8.7136(15)$ & $0.26122(10)$ & & & \\
\hline$e^{+}-\mathrm{Be}\left({ }^{3} P\right)$ & $2.326(6)$ & $4.148(10)$ & $9.55(8)$ & $8.78(3)$ & $8.710(10)$ & $0.2633(5)$ & $0.0000016(5)$ & $0.00505(7)$ & $1.0170(150)$ \\
\hline
\end{tabular}



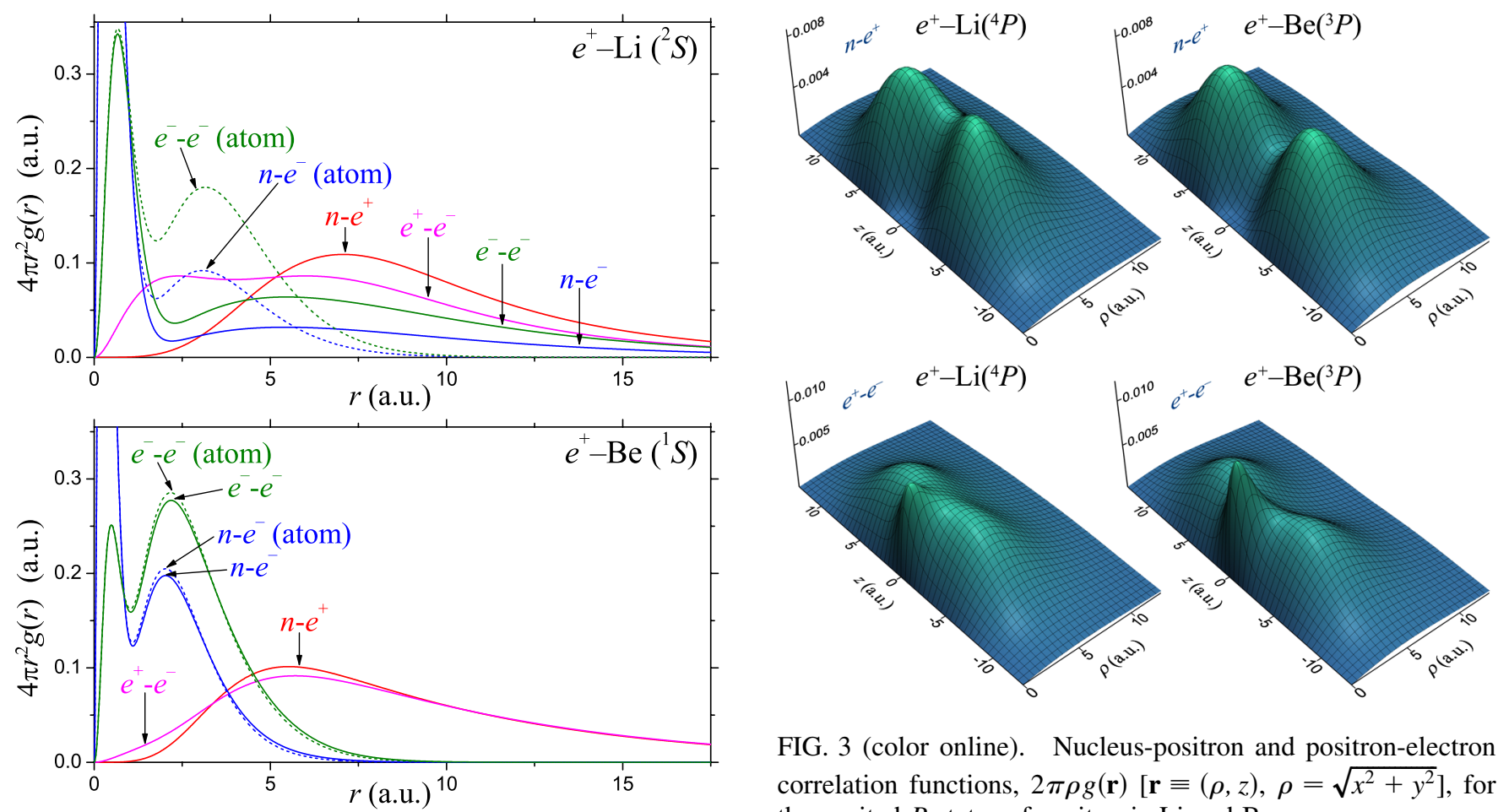

FIG. 3 (color online). Nucleus-positron and positron-electron correlation functions, $2 \pi \rho g(\mathbf{r})\left[\mathbf{r} \equiv(\rho, z), \rho=\sqrt{x^{2}+y^{2}}\right]$, for the excited $P$ states of positronic $\mathrm{Li}$ and $\mathrm{Be}$.

FIG. 2 (color online). Pair correlation functions for the ground $S$ states of positronic lithium and beryllium. Dashed lines correspond to isolated atoms with no positron.

Interestingly, the positron-electron correlation function for the $P$ state of positronic lithium also has a noticeable (albeit shorter) peak at small distances. Therefore, there should be certain admixture of Ps- $\mathrm{A}^{+}$configuration in this state as well. Nucleus-positron correlation functions for the $P$ states of both $e^{+}$- $\mathrm{Li}$ and $e^{+}$-Be presented in Fig. 3 do not have a node (only a concavity) at $z=0$. The absence of the node suggests that the positron is in a mixture of states with zero and nonzero orbital angular momenta.

In Table III we also show the expectation values of the two-particle delta functions. Knowledge of the average electron-positron densities at coalescence points allows us to predict the lifetimes against annihilation. The total spin-averaged particle-antiparticle annihilation rate (see Ref. [33] for details) is given by

$\Gamma=\pi \frac{\alpha^{4} c}{a_{0}}\left[1+\left(\frac{19 \pi}{12}-\frac{17}{\pi}\right) \alpha-2 \alpha^{2} \ln \alpha\right] N_{e^{+}} N_{e^{-}}\left\langle\delta_{e^{+} e^{-}}\right\rangle$,

where $\alpha$ is the fine structure constant, $c$ is the speed of light, $a_{0}$ is the Bohr radius, and $N_{e^{+}}$and $N_{e^{-}}$are the number of positrons and electrons in the system. The terms following the unity in the square brackets of Eq. (3) represent the leading radiative corrections $[34,35]$.

It is important to note that the annihilation rates in the ground and excited states of both $e^{+}-\mathrm{Li}$ and $e^{+}$-Be are significantly different. In the case of lithium, $\Gamma$ is about 3

times smaller in the excited ${ }^{4} P^{o}$ state than in the ground ${ }^{2} S$ state, $1.7484(30) \times 10^{9} \mathrm{~s}^{-1}$ vs $0.59511(150) \times 10^{9} \mathrm{~s}^{-1}$, respectively. For beryllium, the situation is opposite. The ground state survives for roughly twice longer than the excited state: $\Gamma\left({ }^{1} S\right)=0.43095(200) \times 10^{9} \mathrm{~s}^{-1}$, while $\Gamma\left({ }^{3} P^{o}\right)=1.0170(150) \times 10^{9} \mathrm{~s}^{-1}$. The lower electronpositron annihilation rate in $e^{+}-\operatorname{Li}\left({ }^{4} P^{o}\right)$ and $e^{+}-\operatorname{Be}\left({ }^{1} S\right)$ should be attributed to the fact that in these states the positron is alone and only weakly interacts with the atom. Hence, the electron and positron components of the wave function overlap little. In contrast, the most significant configuration in $e^{+}-\mathrm{Li}\left({ }^{2} S\right)$ and $e^{+}-\mathrm{Be}\left({ }^{3} P^{o}\right)$ is Ps-A. The electron in the Ps atom is the one which most likely annihilates with the positron. A substantial difference in $\Gamma$ of the ground and excited states should be a welcome fact for possible spectroscopic studies that detect optically induced changes in the annihilation yield.

To summarize, we have investigated positron-atom complexes in excited states $e^{+}-\mathrm{Li}\left(1 s 2 s 2 p^{4} P^{o}\right)$ and $e^{+}-\mathrm{Be}$ $\left(1 s^{2} 2 s 2 p^{3} P^{o}\right)$. Based on the results of variational calculations with all-particle correlated basis sets, we have obtained a rigorous confirmation that both of these states should be dynamically stable. The $1 s^{2} 2 s 2 p^{3} P^{o}$ state has the advantage of being the lowest excited state of Be and having a long (exceeding seconds) lifetime against radiative decay. Theoretical evidence for the capability of twoto four-electron atoms to form excited states with positrons obtained in Refs. $[9,15,16]$ as well as in this work raise the question as to whether positron binding in excited states should be of common occurrence. Indeed, while the ground state of an atom may not always possess the properties 
necessary for attaching a positron, it is more likely that suitable candidates (e.g., those with higher polarizability and ionization potential close to 0.25 hartree) can be found among the multitude of excited states. Routine search for weakly bound positron-atom states, however, remains a difficult task and will require the development of new, reliable, and computationally inexpensive approaches in the future. We hope that the conclusive evidence for the existence of a weakly bound excited state of positronic beryllium may stimulate future experimental efforts to perform spectroscopic measurements that involve the ground to excited state transition in this and other similar systems.

The authors gratefully acknowledge financial support from the NSF, Grant No. CHE-1300118.

[1] G. G. Ryzhikh and J. Mitroy, Phys. Rev. Lett. 79, 4124 (1997).

[2] G. G. Ryzhikh, J. Mitroy, and K. Varga, J. Phys. B 31, 3965 (1998).

[3] K. Varga, Phys. Rev. Lett. 83, 5471 (1999).

[4] Z.-C. Yan and Y.K. Ho, Phys. Rev. A 59, 2697 (1999).

[5] J. Mitroy, M. W. J. Bromley, and G. G. Ryzhikh, J. Phys. B 35, R81 (2002).

[6] M. W. J. Bromley and J. Mitroy, Phys. Rev. Lett. 97, 183402 (2006).

[7] D. M. Schrader, in Physics with Many Positrons, Proceedings of the International School of Physics "Enrico Fermi" Vol. 174, edited by A. Dupasquier, A. Mills, and R. Brusa (IOS Press, Amsterdam, Netherlands, 2010), p. 77.

[8] X. Cheng, D. Babikov, and D. M. Schrader, Phys. Rev. A 83, 032504 (2011).

[9] D. Bressanini, Phys. Rev. Lett. 109, 223401 (2012).

[10] V. A. Dzuba, V. V. Flambaum, and G. F. Gribakin, Phys. Rev. Lett. 105, 203401 (2010).

[11] G. F. Gribakin, J. A. Young, and C. M. Surko, Rev. Mod. Phys. 82, 2557 (2010).

[12] C. M. Surko, J. R. Danielson, G.F. Gribakin, and R. E. Continetti, New J. Phys. 14, 065004 (2012).
[13] D. B. Cassidy, T. H. Hisakado, H.W. K. Tom, and A.P. Mills, Phys. Rev. Lett. 108, 133402 (2012).

[14] K. Varga, J. Usukura, and Y. Suzuki, Phys. Rev. Lett. 80, 1876 (1998).

[15] G. Ryzhikh and J. Mitroy, J. Phys. B 31, 3465 (1998).

[16] M. W. J. Bromley, J. Mitroy, and K. Varga, Phys. Rev. Lett. 109, 063201 (2012).

[17] J. Mitroy and J. Grineviciute, Phys. Rev. A 88, 022710 (2013).

[18] Y. K. Ho, Phys. Rev. A 41, 68 (1990).

[19] M. W. J. Bromley and J. Mitroy, Phys. Rev. A 75, 042506 (2007).

[20] M. Levitt, R. Novick, and P. D. Feldman, Phys. Rev. A 3, 130 (1971).

[21] C. F. Fischer and G. Tachiev, At. Data Nucl. Data Tables 87, 1 (2004).

[22] M. W. J. Bromley and J. Mitroy, Phys. Rev. A 66, 062504 (2002).

[23] K. Strasburger and H. Chojnacki, in Explicitly Correlated Wave Functions in Chemistry and Physics, Progress in Theoretical Chemical Physics Vol. 13, edited by J. Rychlewski (Springer, Netherlands, 2003), p. 439.

[24] M. Hamermesh, Group Theory and Its Application to Physical Problems (Addison-Wesley, Reading, MA, 1962).

[25] Y. Suzuki and K. Varga, Stochastic Variational Approach to Quantum-Mechanical Few-Body Problems, Lecture Notes in Physics (Springer, Berlin, 1998).

[26] S. Bubin, M. Pavanello, W.-C. Tung, K. L. Sharkey, and L. Adamowicz, Chem. Rev. 113, 36 (2013).

[27] J. Mitroy, S. Bubin, W. Horiuchi, Y. Suzuki, L. Adamowicz, W. Cencek, K. Szalewicz, J. Komasa, D. Blume, and K. Varga, Rev. Mod. Phys. 85, 693 (2013).

[28] S. Bubin, O. V. Prezhdo, and K. Varga, Phys. Rev. A 87, 054501 (2013).

[29] A. E. Kramida, Yu. Ralchenko, J. Reader, and NIST ASD Team, NIST Atomic Spectra Database 5.1, http:// physics.nist.gov/asd (2013).

[30] J. Mitroy, Phys. Rev. A 70, 024502 (2004).

[31] J. Mitroy, J. At. Mol. Sci. 1, 275 (2010).

[32] J. Mitroy, Phys. Rev. Lett. 94, 033402 (2005).

[33] S. Bubin and K. Varga, Phys. Rev. A 84, 012509 (2011).

[34] I. Harris and L. M. Brown, Phys. Rev. 105, 1656 (1957).

[35] I. Khriplovich and A. Yelkhovsky, Phys. Lett. B 246, 520 (1990). 